\title{
Computational challenges in high angle of attack flow prediction
}

\author{
Russell M. Cummings ${ }^{\mathrm{a}}$, James R. Forsythe ${ }^{\mathrm{b}}$, Scott A. Morton ${ }^{\mathrm{b}}$, Kyle D. Squires ${ }^{\mathrm{c}}$ \\ a Aerospace Engineering Department, California Polytechnic State University, San Luis Obispo, CA 93407, USA \\ ${ }^{\mathrm{b}}$ Department of Aeronautics, United States Air Force Academy, USAF Academy, CO 80840, USA \\ ${ }^{\mathrm{c}}$ Department of Mechanical and Aerospace Engineering, Arizona State University, Tempe, AZ 85287, USA
}

\begin{abstract}
Aircraft aerodynamics have been predicted using computational fluid dynamics for a number of years. While viscous flow computations for cruise conditions have become commonplace, the non-linear effects that take place at high angles of attack are much more difficult to predict. A variety of difficulties arise when performing these computations, including challenges in properly modeling turbulence and transition for vortical and massively separated flows, the need to use appropriate numerical algorithms if flow asymmetry is possible, and the difficulties in creating grids that allow for accurate simulation of the flowfield. These issues are addressed and recommendations are made for further improvements in high angle of attack flow prediction. Current predictive capabilities for high angle of attack flows are reviewed, and solutions based on hybrid turbulence models are presented.
\end{abstract}

\section{Contents}

1. Introduction . . . . . . . . . . . . . . . . . . . . . . . . 370

2. Computational challenges . . . . . . . . . . . . . . . . . . . 372

2.1. Governing equation complexity . . . . . . . . . . . . . . . 372

2.2. Turbulence modeling . . . . . . . . . . . . . . . . . . . . . . . . 374

2.3. Transition modeling . . . . . . . . . . . . . . . . . . . . 376

2.4. Flowfield asymmetry and algorithm symmetry . . . . . . . . . . . . . . . . . . . 377

2.5. Grid generation and density . . . . . . . . . . . . . . . . . . . . . 379

2.6. Numerical dissipation . . . . . . . . . . . . . . . . . . . . . . 380

3. Computational results and future directions . . . . . . . . . . . . . . . . . . . . . 381

4. Conclusions . . . . . . . . . . . . . . . . . . . . . . . . . 382

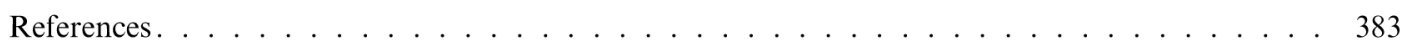




\section{Introduction}

Aircraft fly at a variety of incidence angles, depending on their purpose and flight requirements. For instance, commercial transports rarely fly at an angle of attack larger than $\alpha=10^{\circ}$, but tactical aircraft and missiles fly regularly at angles of attack above $\alpha=20^{\circ}$. During unsteady maneuvers, such as the Cobra maneuver performed by the Su-27, aircraft may even fly at angles of attack over $\alpha=90^{\circ}$. Key geometric components of aircraft while flying at high angles of attack are forebodies, wings, and strakes (or leading-edge extensions); each of these creates special difficulties when attempting to model the flowfield.

While it is somewhat difficult to precisely describe the various angle of attack regions, a good categorization system has been developed [1-4]:

- low angle of attack $0^{\circ} \leqslant \alpha \leqslant 15^{\circ}$ (attached, symmetric, steady flow, linear lift variation);
- medium angle of attack $15^{\circ} \leqslant \alpha \leqslant 30^{\circ}$ (separated, symmetric rolled-up vortices, steady flow, non-linear lift variation);

- high angle of attack $30^{\circ} \leqslant \alpha \leqslant 65^{\circ}$ (separated, asymmetric rolled-up vortices, steady/unsteady flow, nonlinear lift variation);

- very high angle of attack $\alpha>65^{\circ}$ (separated, unsteady turbulent wake, post stall).

These angle of attack regimes cannot be defined precisely, since the aircraft geometry and freestream conditions will create conditions where flow of one type will exist at angles of attack outside the ranges given. A fairly good representation of the regimes is shown in Fig. 1.

An example of an axisymmetric slender body at medium angle of attack is shown in Fig. 2. The flow separates in the crossflow plane and wraps up into two, counter-rotating, symmetric vortices. These vortices then lift away from the fuselage, and new vortices begin
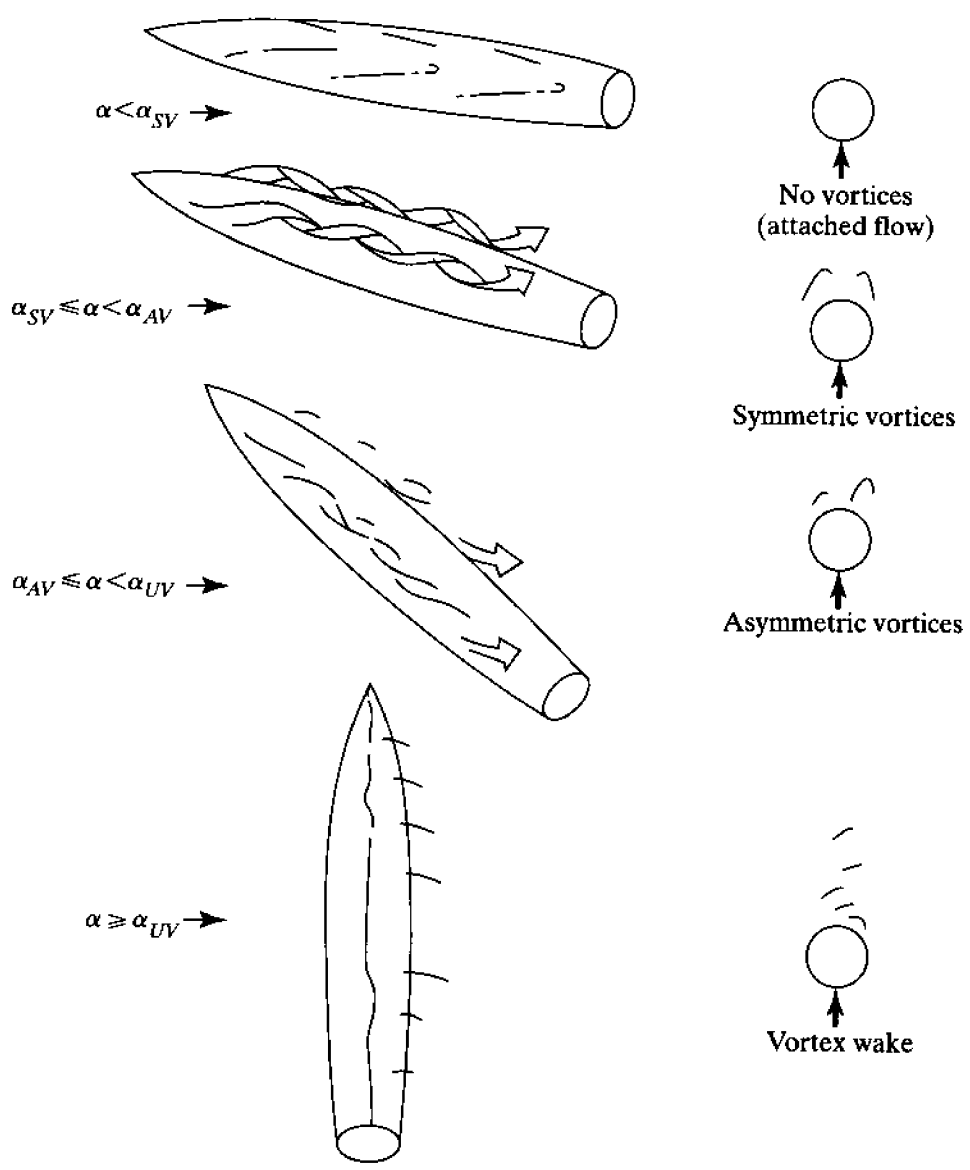

Fig. 1. Angle of attack flow regimes ( $\alpha_{\mathrm{SV}}$ is the angle of attack where symmetric vortices are formed, $\alpha_{\mathrm{AV}}$ is the angle of attack where asymmetric vortices are formed, and $\alpha_{\mathrm{UV}}$ is the angle of attack where an unsteady vortex wake is formed; from [1-4]). 


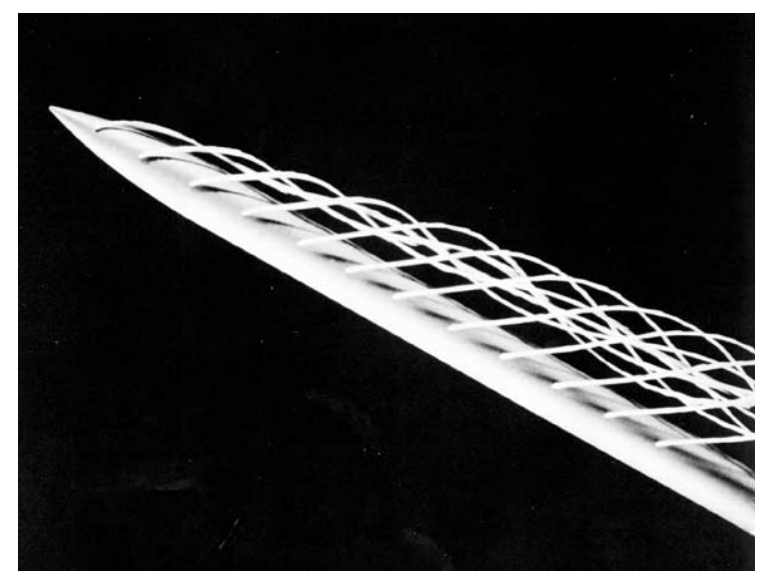

Fig. 2. Slender body at medium angle of attack $\alpha=30^{\circ}$ (from [5]).

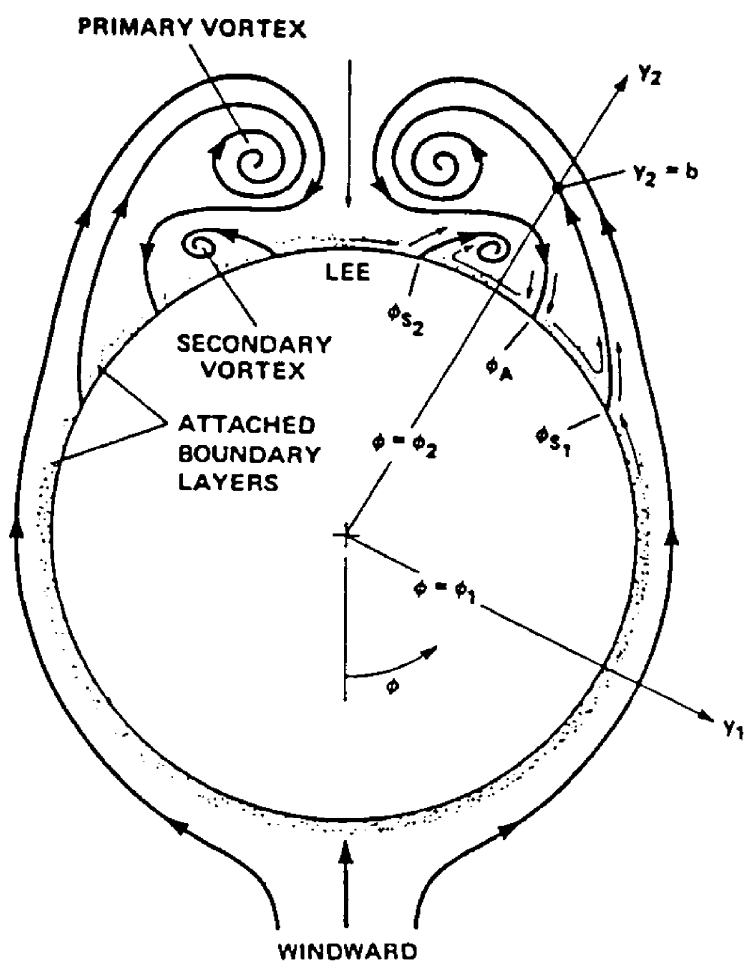

Fig. 3. Slender body flow topology at medium angles of attack (from [6]).

to form further downstream. The crossflow flowfield topology at one of the downstream crossflow planes is shown in Fig. 3. When the high angle of attack regime is reached, the vortices become asymmetric, creating a flow topology as shown in Fig. 4. Similar flowfields exist above wings at high angles of attack, with the delta wing flowfield serving as a representative case. The medium angle of attack flowfield above a delta wing is shown in Fig. 5, where the vortices are steady and coherent. At

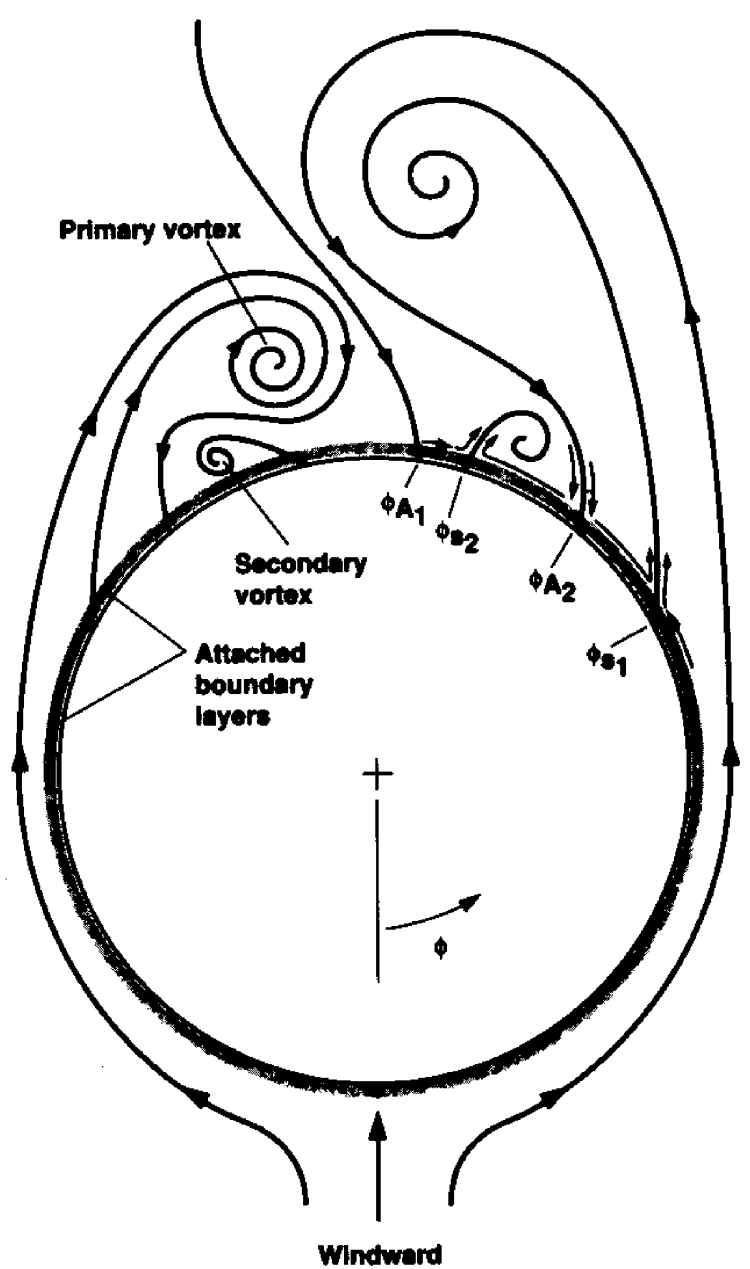

Fig. 4. Slender body flow topology at high angles of attack (from [7]). (C) 1992 by the American Institute of Aeronautics and Astronautics, Inc. Reprinted with permission.

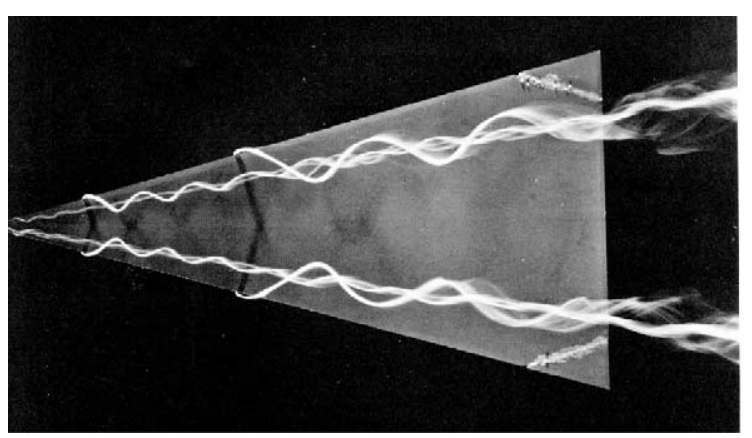

Fig. 5. Delta wing at medium angle of attack (from [5]).

higher angles of attack, the adverse pressure gradient of the wing causes vortex breakdown to take place, yielding an unsteady and highly non-linear flowfield (see Fig. 6). The breakdown location moves forward as the angle of 


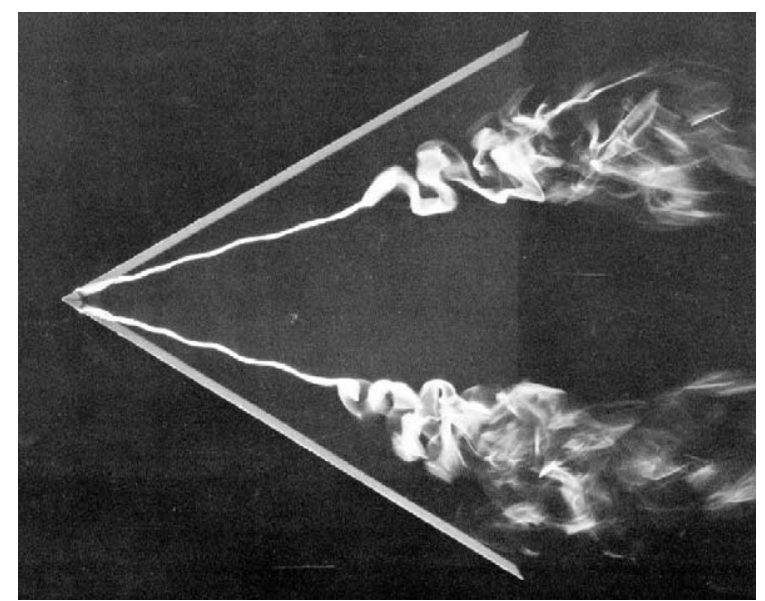

Fig. 6. Delta wing at high angle of attack with vortex breakdown (from [5]).

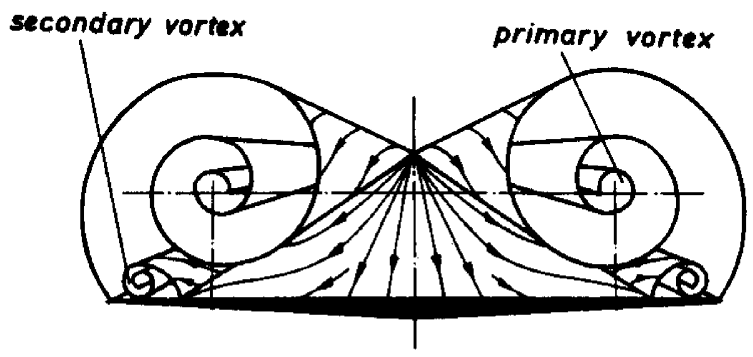

Fig. 7. Delta wing flow topology at medium angle of attack (the original version of this figure was first published by AGARD/NATO in [8]).

attack is increased, further complicating the features of the flowfield. The crossflow flowfield topology for a delta wing at medium angles of attack is shown in Fig. 7.

There are certain similar features in these flowfields. The crossflow approaches the fuselage from the windward side and forms a boundary layer that eventually separates at location $\phi_{S_{1}}$ (see Figs 3 and 4). The separated flow creates a pair of primary vortices that reattach at locations marked $\phi_{A}$. The primary vortices induce an outward flow on the leeside of the fuselage that eventually separates at $\phi_{S_{2}}$ to form secondary vortices. The secondary vortices can also create tertiary vortices, with each set of vortices rotating in opposite directions from the set above them. When the vortices are asymmetric, as shown in Fig. 4, the pressure differences between the left and right side of the fuselage cause a side force on the configuration which can often produce significant yaw moments due to the large moment arm to the forebody or strake.

The flow topology is quite complex with separation points/lines (primary, secondary, and tertiary, etc.), attachment points/lines, and vortex interaction. This viscous-dominated flowfield is highly non-linear and can lead to complex flow topologies, such as vortex breakdown. The resulting flowfields are very difficult to predict theoretically, which leads to the necessity of using experiments and/or computational fluid dynamics (CFD) to model the flow. Researchers in high angle of attack aerodynamics have noted a variety of interesting features of the flow, including [1,2]:

- vortex asymmetry begins when the angle of attack is about twice the nose angle;

- separation is very sensitive to nose geometric asymmetries;

- increased nose bluntness decreases asymmetry; and

- the results are difficult to reproduce experimentally.

\section{Computational challenges}

While all CFD simulations require a detailed knowledge of fluid dynamics, numerical methods, mathematics, and the use of computer systems, high angle of attack aerodynamics has its own set of difficulties that are not always seen in other flowfields. Some of the issues that are extremely important for the accurate simulation of high angle of attack flowfields include:

- governing equation complexity;

- turbulence modeling;

- transition modeling;

- algorithm symmetry;

- grid generation and density;

- numerical dissipation.

While a detailed understanding of each of these issues would require volumes of information and years of experience, a brief overview of the specific difficulties encountered in high angle of attack aerodynamics will be addressed.

\subsection{Governing equation complexity}

The governing equations of fluid dynamics for a Newtonian fluid are the Navier-Stokes equations, which are often written in body-conforming coordinates $(\xi, \eta, \zeta)$ and represented in vector form for ease in applying numerical algorithms

$\partial_{\tau} \hat{Q}+\partial_{\xi}\left(\hat{F}+\hat{F}_{v}\right)+\partial_{\eta}\left(G+\hat{G}_{v}\right)+\partial_{\zeta}\left(\hat{H}+\hat{H}_{v}\right)=0$,

where $\hat{Q}$ represents the vector of conserved quantities (mass, momentum, and energy), $\hat{F}, \hat{G}$, and $\hat{H}$ represent the inviscid flux vectors, and $\hat{F}_{v}, \hat{G}_{v}$, and $\hat{H}_{v}$ are fluxes containing the viscous terms. Details about the equation set and the definition of the various flux vectors may be found in Viviand's article [9].

The Navier-Stokes equations are a set of five, coupled, non-linear, second-order partial differential equations with no known general, closed-form solution. 
There are various techniques for the numerical prediction of turbulent flows, ranging from Reynolds-averaged Navier-Stokes (RANS), to large eddy simulation (LES), to direct numerical simulation (DNS). DNS attempts to resolve all scales of turbulence, from the largest to the smallest, by solving Eq. (1) directly. Because of this, the grid resolution requirements are very high, and increase drastically with Reynolds number - this is only currently possible for low Reynolds number flowfields, such as flat plates, shear layers, and simple three-dimensional geometries [10]. LES attempts to model the smaller, more homogeneous scales, while resolving the larger, energy-containing scales, which makes the grid requirements for LES significantly less than for DNS. To accurately resolve the boundary layer, however, LES must accurately resolve the energy-containing eddies in the boundary layer, which requires very small streamwise and spanwise grid spacing. Finally, the RANS approach attempts to solve the time-averaged flow, which means that all scales of turbulence must be modeled. The RANS equations appear to be identical to the full Navier-Stokes equations (Eq. (1)), although all flow variables have been replaced with time-averaged values. RANS models often fail to provide accurate results for high angle of attack flows since the large turbulence scales for separated flows are very dependent on the geometry. RANS models, however, can provide accurate results for attached boundary layer flows, thin shear layers, and steady coherent vortical flowfields, but at the cost of increasing empiricism due to the closure problem. Spalart provides a good discussion and comparison of these various approaches [11].

These various techniques have very different computational requirements. In 1997, Spalart et al. estimated that LES computations over an entire aircraft would not be possible for over 45 years [12]. Of course, that makes DNS computations for full aircraft unthinkable for the foreseeable future. Spalart's estimate led to the formulation of detached-eddy simulation (DES), which is a hybrid approach combining the advantages of LES and RANS into one model. For the DES approach, RANS is used in the boundary layer, where it performs well (and with much lower grid requirements than LES), and LES is then used in the separated regions where its ability to predict turbulence length scales is important. Shur et al. [13] calibrated the model for isotropic turbulence, and applied it to an NACA 0012 airfoil section; the model agreed well with lift and drag predictions to $90^{\circ}$ angle of attack.

Historically, solutions of the Navier-Stokes equations required a great deal of computer resources, and until recently solutions were only obtainable on supercomputers. Because of the limitations of computers, even the RANS equations were often simplified for the high angle of attack case. One way to simplify the RANS equation set is to assume that the flow is steady and that the longitudinal viscous terms may be neglected (Eq. (2)). This creates a parabolic-hyperbolic equation set that allows for solution by marching longitudinally in space. These assumptions restrict solutions to supersonic flow cases at high Reynolds numbers (thin boundary layers) with no upstream recirculation in the flowfield. The advantage of the parabolized form of the equations is that solutions may be obtained very quickly when compared with the full RANS equations (Eq. (1)) [10]:

$\partial_{\xi} \hat{F}+\partial_{\eta} \hat{G}+\partial_{\zeta} \hat{H}=\frac{1}{R e}\left(\partial_{\eta} \hat{S}+\partial_{\zeta} \hat{S}\right)$.

Another simplification of the RANS equations that has been used is the "thin layer" equations. These equations are derived from Eq. (1) by assuming that only the viscous terms in the surface-normal direction are essential for resolving the flowfield, yielding

$\partial_{\tau} \hat{Q}+\partial_{\xi} \hat{F}+\partial_{\eta} \hat{G}+\partial_{\zeta} \hat{H}=\frac{1}{R e} \partial_{\zeta} \hat{S}$.

Various alternative forms of the thin-layer equations exist, all based on the assumption that various viscous derivatives may be neglected (such as cross derivatives). Eq. (3) is only valid for thin boundary layers, and thus is used for flows at high Reynolds numbers. The equations require considerably more time to compute than Eq. (2), however, because they must be solved by marching in time. Upstream propagation and recirculation, however, are allowed, and thus these equations can be used in subsonic flow with separated regions. Degani and Marcus showed that these equations could adequately resolve steady vortical flow structures in the medium angle of attack range where the vortices were symmetric [14]. Fig. 8a shows the side force coefficient, $C_{Y}$, for an ogive cylinder at $\alpha=40^{\circ}$; the computations were made using both the full Navier-Stokes equations and the thin-layer equations (FNS and TLNS, respectively, in the figure). Little or no difference in the solutions can be seen after a non-symmetric disturbance is added to the flowfield at approximately $135 \mathrm{~s}$. At high angles of attack, however, they found that differences existed between Eqs. (1) and (3) for the same flow conditions and geometry, and that the differences increased with angle of attack, as shown in Fig. 8b. Degani and Marcus conjectured that the two equation sets were following different paths of asymmetric vortex formation once the disturbance had been initiated. The thin-layer equations, however, should probably only be used for steady flow at medium to high angles of attack in order to maintain the assumptions the equations are based on.

Finally, the equation set can be simplified further by assuming that the flowfield is inviscid, yielding Euler's equation

$\partial_{\tau} \hat{Q}+\partial_{\xi} \hat{F}+\partial_{\eta} \hat{G}+\partial_{\zeta} \hat{H}=0$.

Euler's equation cannot be used to predict separation. However, in cases where the separation location is 


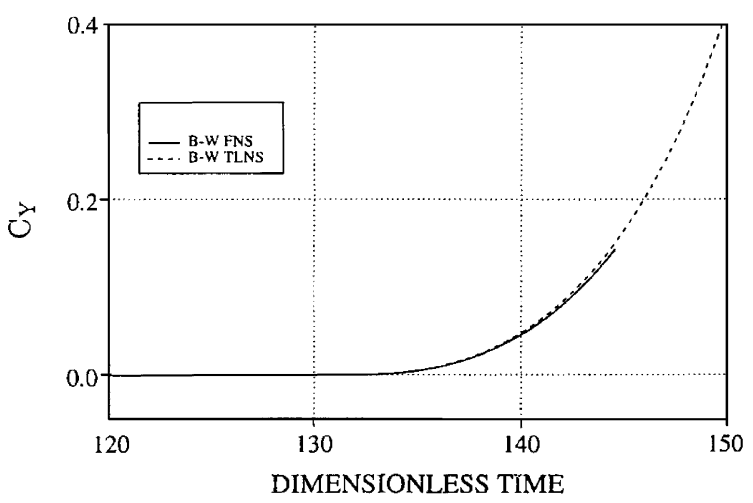

(a)

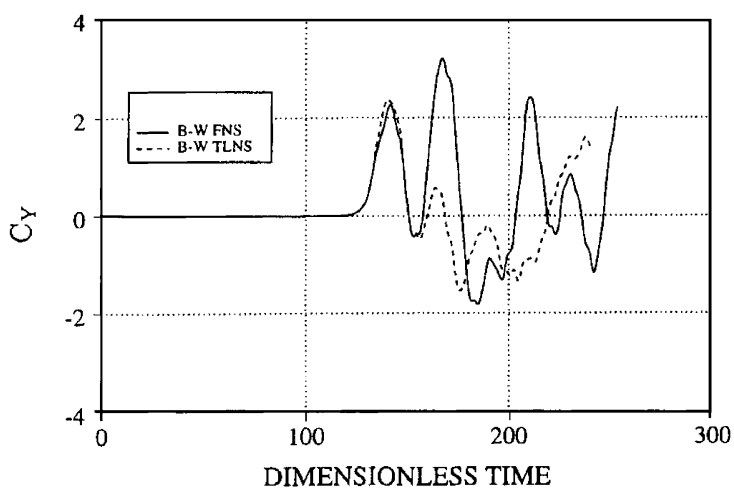

(b)

$$
\alpha=60^{\circ}
$$

Fig. 8. Time history of side-force coefficient (from [14]); $\mathrm{B}-\mathrm{W}=$ body-wing,$\quad \mathrm{FNS}=$ full $\quad$ Navier-Stokes $\quad$ (RANS), TLNS $=$ thin-layer Navier-Stokes.

known a priori, the equations can be used to compute the vortical flowfield. The most common application of Euler's equation to high angle of attack aerodynamics is for delta wing configurations. Delta wings with sharp leading edges have a fixed primary vortex separation location, and the equations do a reasonable job in simulating the linear characteristics of the flowfield, but cannot model the non-linear interaction of secondary or tertiary vortices on the location and strength of the primary vortices. Configurations without fixed separation locations cannot be handled well using Euler's equation; most practical aircraft configurations would make it difficult to obtain good high angle of attack flow simulations using the Euler equations.

\subsection{Turbulence modeling}

The RANS form of the Navier-Stokes equations is used in many practical high angle of attack applications to reduce the computational time and memory required for performing non-averaged computations. While DNS Navier-Stokes calculations are being performed on increasingly complex geometries, these geometries are still relatively basic and can be solved only at very low Reynolds numbers. Because of these restrictions, the Navier-Stokes equations have been Reynolds (time or ensemble) averaged [15]. For compressible flows the equations are Favre (mass-weighted) averaged [16]. The averaging process introduces correlations between fluctuating flow variables (the Reynolds stresses, Eq. 5) that require the use of a turbulence model in order to affect closure of the equation set

$\tau_{i j}=-\rho \overline{u_{i}^{\prime} u_{j}^{\prime}}$.

Turbulence models are semi-empirical formulations that are used to close the RANS equations by providing the Reynolds stresses. They are generally calibrated on building block flows such as boundary layers, shear layers, and wakes [17]. The Reynolds stresses are modeled in one of two ways: either through an eddyviscosity model or a stress-transport model. Stress transport models make no general assumptions about the form of the six components of the Reynolds stress. Unless additional assumptions are made, these models are therefore solving for six unknowns. The more common eddy-viscosity models are based on the Boussinesq approximation-that the Reynolds stresses are proportional to the strain rate of the mean flow. The turbulent eddy viscosity $\left(\mu_{t}\right)$ is the constant of proportionality, i.e.

$-\rho \overline{u_{i}^{\prime} u_{j}^{\prime}}=\mu_{\mathrm{t}}\left(\frac{\partial u_{i}}{\partial x_{j}}+\frac{\partial u_{j}}{\partial x_{i}}\right)$.

This assumption reduces the number of unknowns from the six components of the Reynolds stresses to a single unknown, the turbulent eddy viscosity. Because Eq. (6) takes the same form as the laminar stresses, the turbulent eddy viscosity can simply be added to the laminar viscosity in the Navier-Stokes equation, i.e. $\mu=$ $\mu_{1}+\mu_{\mathrm{t}}$ ( $\mu_{1}$ is the laminar viscosity and $\mu_{\mathrm{t}}$ is the turbulent eddy viscosity). This is the reason Eq. (1) appears similar for both the DNS and RANS form of the Navier-Stokes equations. In addition, all flow variables are replaced by their time-averaged values (e.g. $u_{i}$ is replaced by $\bar{u}_{i}$ ). The turbulent viscosity is then provided by the turbulence model, which is often classified by the number of partial differential equations it adds. Most common are zero-, one-, and two-equation turbulence models. The zeroequation models use algebraic relationships rather than a partial differential equation. Since the modeled equations are semi-empirical, they require experimentally determined coefficients that are usually found for flow over simple geometries like a flat plate or various types of shear layers.

For compressible flows additional terms similar to Reynolds stresses appear in the energy equation involving correlations of fluctuating velocities and temperature-the turbulent heat flux vector. These terms 
account for enhanced heat transfer due to the turbulent motions, and are commonly modeled by appealing to the Reynolds analogy which relates the heat transfer to the momentum transfer by the Prandtl number. By assuming a turbulent Prandtl number (generally assumed constant), the turbulent heat transfer can therefore be obtained without any additional equations. See Ref. [18] for a more complete discussion.

But what happens at high angles of attack? Do the turbulence models adequately resolve the flow features found in separated, vortical flowfields? An illustration of the difficulties can be seen by using the zero-equation (algebraic) turbulence model of Baldwin and Lomax [19] as an example. The Baldwin-Lomax model contains a function, $F(y)$, which is used as part of the outer-layer model

$F(y)=y \Omega\left[1-\mathrm{e}^{-\left(y^{+} / A^{+}\right)}\right]$,

where $y$ is the normal distance to a flat plate and $\Omega$ is the vorticity magnitude. In an attached boundary layer (see Fig. 3, $\phi=\phi_{1}$ radial line), $F(y)$ increases to a maximum value and then decreases near the edge of the boundary layer. However, in a separated vortical flow layer (see Fig. 3, $\phi=\phi_{2}$ radial line), $F(y)$ attains a local maxima in the attached boundary layer and then reaches a global maxima in the separated layer (see Fig. 9). The turbulence model chooses the highest $F(y)$ and its corresponding distance from the wall, which greatly overpredicts the turbulent viscosity in this region. The overpredicted turbulent viscosity creates non-physical results when added to the laminar viscosity, altering separation locations and the proper formation of secondary and tertiary vortices.

A variety of researchers have proposed methods for altering algebraic turbulence models for high angle of attack flow [6,20,21]. Degani and Schiff proposed a modification to the model (and hence to all eddyviscosity turbulence models) that obtains the correct

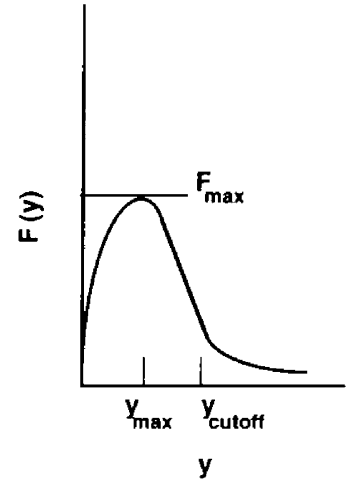

(a) Windward side

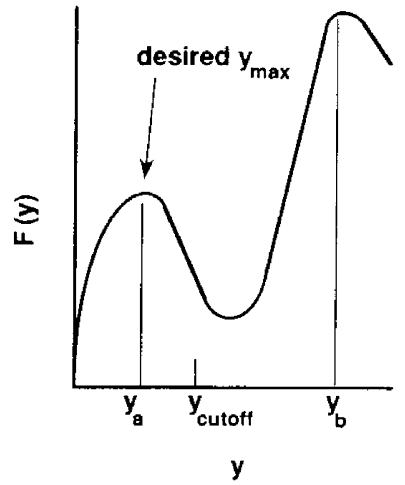

(b)
Leeward side
Fig. 9. Variation of Baldwin-Lomax outer layer function, $F(y)$ (from [6]). value of $F(y)$ and gives better prediction of the flow topology in a separated flow region [6]. This modification has led to the ability to accurately simulate steady vortical flows with RANS computations. An example of the improvements to both zero-equation and oneequation turbulence models for predicting vortical flowfields was done by Gee et al. [22]. Vortical flow modifications for the $k-\varepsilon$ turbulence model have also been suggested [23] and have been applied to flow over slender bodies at high incidence angles [24]. However, in spite of these improvements the RANS-based equations still lead to questionable predictions at high to very high angles of attack, where the flow is unsteady and the time-averaged equations are no longer capable of properly modeling the flowfield. This has led to further developments in equation and turbulence modeling, including vortex filtering [25] and the DES method.

DES was proposed by Spalart et al. [11,12] as a method to combine the best features of LES with the best features of the RANS approach. RANS tends to be able to predict attached flows very well with a relatively low computation cost. LES, on the other hand, has a high computation cost, but can predict unsteady separated flows more accurately. The model was originally based on the Spalart-Allmaras one-equation RANS turbulence model [26]. The wall destruction term is proportional to $\tilde{v} / d^{2}$, where $d$ is the distance to the closest wall. When this term is balanced with the production term, the eddy viscosity becomes $\tilde{v} \propto S d^{2}$, where $S$ is the local strain rate. The Smagorinski LES model [27] varies the sub-grid-scale (SGS) turbulent viscosity with the local strain rate and the grid spacing, $\Delta$ (i.e. $v_{\mathrm{SGS}} \propto S \Delta^{2}$ ). If, therefore, $d$ is replaced by $\Delta$ in the wall destruction term, the Spalart-Allmaras model will act as a Smagorinski LES model.

To exhibit both RANS and LES behavior, $d$ in the Spalart-Allmaras model is replaced by $\tilde{d}=$ $\min \left(d, C_{\mathrm{DES}} \Delta\right)$, where $C_{\mathrm{DES}}$ is the DES model constant. When $d=\Delta$, the model acts as a RANS model, and when $d \ll \Delta$, the model acts as a Smagorinski LES model. Therefore, the model can be "switched" to LES mode by locally refining the grid. In an attached boundary layer, a RANS simulation will have highly stretched grids in the streamwise direction. To retain RANS behavior in this case, $\Delta$ is taken as the largest spacing in any direction $(\Delta=\max (\Delta x, \Delta y, \Delta z))$. This type of extension can be applied to other turbulence models as well, as has been shown by Strelets [28] and Forsythe et al. [29]. The DES approach provides a way to model unsteady, asymmetric flowfields at high to very high angles of attack without resorting to new and untrustworthy "fixes" to flat-plate turbulence models.

DES has the advantage of computing the unsteady three-dimensional flow features necessary to accurately predict flow quantities in massively separated flows. The additional computational cost of DES can be attributed 
to the need to compute a time-accurate flowfield (i.e., it may require more solutions or time steps), as well as a need to accurately resolve small three-dimensional flow structures spatially (i.e., it may require more grid points).

\subsection{Transition modeling}

Most high angle of attack computations are performed under either fully laminar or fully turbulent conditions, with no attempt to model transition. Note that in the present context, "fully laminar" implies a solution of the Navier-Stokes equations in which no explicit turbulence model is included in the calculation. "Fully turbulent" solutions imply that the turbulence model is everywhere active within the boundary layers formed over solid surfaces. Such solutions are established in Reynolds-averaged methods that employ the Boussinesq approximation, for example, by specifying at the inflow boundary a small level of eddy viscosity, sufficient to ignite the model as the fluid enters the boundary layer. Fully laminar or fully turbulent computations are limiting cases but represent the norm in practice since the numerical prediction of laminar-toturbulent transition and application of transition models within large-scale CFD computations remains difficult. Such approaches introduce uncertainty since researchers often compute both fully laminar and fully turbulent solutions and then compare with experimental data. One complication introduced by such an approach is that the amount of transitional flow present in the experiment is often unknown, in turn complicating interpretation of CFD results against measurements.

Further, in many applications of practical importance, laminar-to-turbulent transition can have a crucial effect on the overall behavior of the flow, substantially altering forces and moments. One example is provided by a notional forebody in a crossflow, shown in Fig. 10. The forebody cross section is a rounded-corner square,

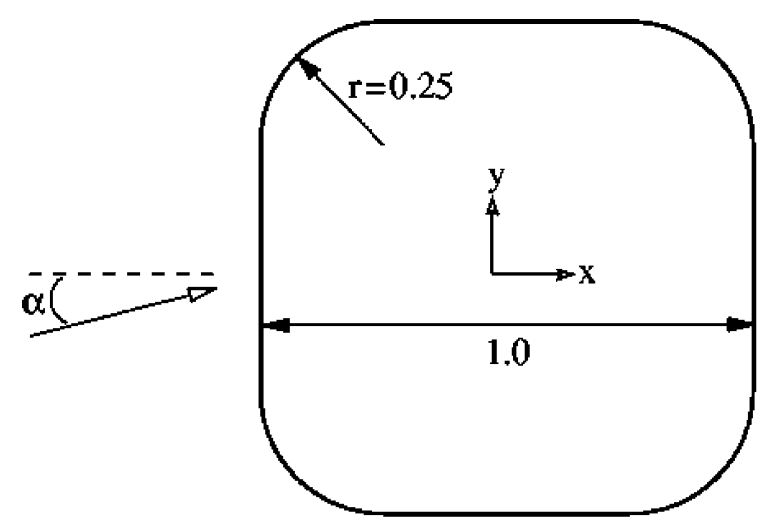

Fig. 10. Cross section of notional rounded-corner forebody. similar to the cross sections of the X-29 and T-38. The flow around the cross section was measured by Polhamus et al. [30] for a range of Reynolds numbers and angles of attack. The motivation was to understand spin characteristics of aircraft forebodies, the angle of attack being idealized to represent an actual aircraft in a flat spin.

The effect of laminar-to-turbulent transition is crucially important for the forebody shown in Fig. 10, as it alters the locations at which boundary layer separation occurs, which in turn affects the streamwise and lateral (side) forces acting on the body. Polhamus et al. found that the side force reverses from positive (along the positive $y$-axis in Fig. 10) to negative at a critical Reynolds number, analogous to the drag crisis which occurs over cylinders and spheres. A reversal of the side force is important since at sub-critical Reynolds numbers the negative side force is spin-propelling, while the positive side force at higher Reynolds numbers is spin-damping. Reversal of the side force is influenced by the location of boundary layer separation along the upper surface of the forebody, which in turn is sensitive to the location of laminar-to-turbulent transition.

Super-critical regimes can be accurately modeled via prediction of the fully turbulent flow. Squires et al. [31] applied DES to prediction of the three-dimensional flow around the forebody shown in Fig. 10, obtaining accurate predictions of the pressure distribution and averaged streamwise and side forces at a Reynolds number of 800,000 , above the critical value. Prediction of the sub-critical flows requires an approach for handling the effect of laminar-to-turbulent transition. One approach is the "tripless" method employed by Travin et al. [32] used for prediction of the sub-critical flow over a circular cylinder. These investigators applied DES, with the baseline closure being based on the Spalart-Allmaras model. Effects of laminar-to-turbulent transition were modeled by seeding the initial condition with a small level of eddy viscosity, and with the level of eddy viscosity at the inlet boundary equal to zero. Once the flow attains equilibrium in the attached regions of the flow (prior to boundary layer separation), the eddy viscosity is zero and the boundary layers are effectively laminar. Recirculation of the flow in the wake of the cylinder provided a mechanism for sweeping non-zero values of the eddy viscosity from downstream to upstream. In this case the turbulence model is sustained by the recirculating motion of the wake, the turbulent region of the flow beginning downstream of separation. While the tripless approach does not attempt to mimic the very complex details governing transition, the method possesses the substantial advantage that the location of transition (identified by the region over which the eddy viscosity sharply increases from zero) is dictated by the turbulence model and flow conditions, rather than the initial and/or boundary conditions. 
An additional example illustrating some of the complexities introduced by details of the process of transition on the interpretation of flowfield predictions is provided by a forebody at angle of attack, for which a crossflow is established as shown in Fig. 2. For the configuration shown, transition can take place along streamlines that convect along the windward plane of symmetry before flowing around the fuselage - what is the laminar run for such a flowfield? Very different results are obtained if the flow attaches within a crossflow plane and then flows around the vehicle than if the flow travels down the length of the forebody (or some partial length of the forebody) and then flows around the vehicle.

A good review of transition models has been performed [33], and various researchers have attempted to apply transition models to CFD computations $[34,35]$. The difficulty with transition modeling is similar to the difficulties with turbulence modeling: most models are either theoretical or semi-empirical and are formulated for flat plates or curved surfaces, but do not possess the breadth of development to support high angle of attack flow predictions. These methods often require the solution of stability equations, which also increases the total computation time for a solution (again, similar to turbulence models). It is doubtful that transition models will be accurate enough to be used in high-angle flow simulations for the foreseeable future; useful modeling of transition should be targeted as a pacing item for full aircraft simulations.

\subsection{Flowfield asymmetry and algorithm symmetry}

As more researchers have simulated medium, high, and very high angle of attack flowfields, a controversy has developed regarding vortex flow asymmetries. As anyone who works in aircraft or missile aerodynamics knows, side forces and yaw moments develop at high angles of attack due to vortex asymmetries on "real-life" configurations. The cause of the asymmetries, however, is not well understood.

Two possible explanations have surfaced for the vortex asymmetry: (1) the asymmetry is due to an absolute hydrodynamic instability - small perturbations yield a bifurcated asymmetry, even after the perturbation is removed (example: the Karman vortex street behind the flow over a cylinder), or (2) the asymmetry is due to a convective instability-small, permanent perturbations are required for asymmetry to exist, and the flowfield is not limited to the two bifurcated states. A good overview of the two views, including references for supporting simulations and theoretical concepts was reported by Thomas [36].

In either case, however, a perturbation is required! In experiments the perturbation is always present, but rarely the same, due to flowfield angularity, freestream turbulence, or surface imperfections on the model. But, what causes the perturbation in numerical calculations? Certainly, a variety of usual suspects can be rounded up, including truncation error, round-off error, the numerical algorithm, boundary conditions, or initial conditions. Everyone agrees that the flow asymmetry in a very high angle of attack flowfield is caused by an absolute instability, but the asymmetries in the high angle of attack region are more difficult to understand, and therefore, to accurately predict.

Zilliac et al. conducted an experiment using a rotatable ogive tip on the front of a cylindrical crosssection afterbody [37]. The results, presented in Fig. 11, showed some very interesting features. At medium angles of attack $\left(\alpha=20^{\circ}\right)$ there was no asymmetry, as was expected. In the high angle of attack range $\left(20^{\circ}<\alpha<50^{\circ}\right)$, the side force coefficient varied continuously with the ogive roll angle - it was possible to obtain any value of side force between the positive and negative maximums. Eventually, as the angle of attack reached the very high range $\left(50^{\circ}<\alpha<65^{\circ}\right)$, the flowfield reached the absolute instability case, with side forces of either a positive or negative maximum, but with no intermediate values. These results suggest that the high angle of attack regime could attain any level of side force between the maxima, a situation that seems to suggest that the asymmetry is due to a convective instability. These results have been verified, with attention paid to the affects of transition caused by various levels of surface roughness [38].

The prediction of these high angle of attack flows was made more difficult to understand since not all researchers performing numerical simulations were replicating these experimental results. Many researchers were computing asymmetric vortices in the high angle of attack range, and using the results as evidence that the asymmetric flowfield was due to an absolute flow instability. Other researchers, however, were computing symmetric vortices for similar geometries at the same angles of attack. As can be imagined, this led to a great deal of disagreement and confusion. In an important study, Levy et al. showed that certain numerical algorithms break symmetry preservation, thus causing the flowfield to be asymmetric [39]. They first investigated the Beam-Warming algorithm [40], which is an implicit algorithm that requires block tridiagonal matrix inversions. The algorithm has relatively high memory requirements and high operation counts per grid point. When computing geometries in the high angle of attack range, the Beam-Warming algorithm produced symmetric flowfields, and the algorithm was proven to be symmetric.

In order to compute flowfields at a variety of subsonic and supersonic Mach numbers, many researchers began using flux-vector splitting algorithms [41]. These algorithms are also implicit and require block tridiagonal matrix inversion, and also have high memory requirements and high operation count per grid point. These 

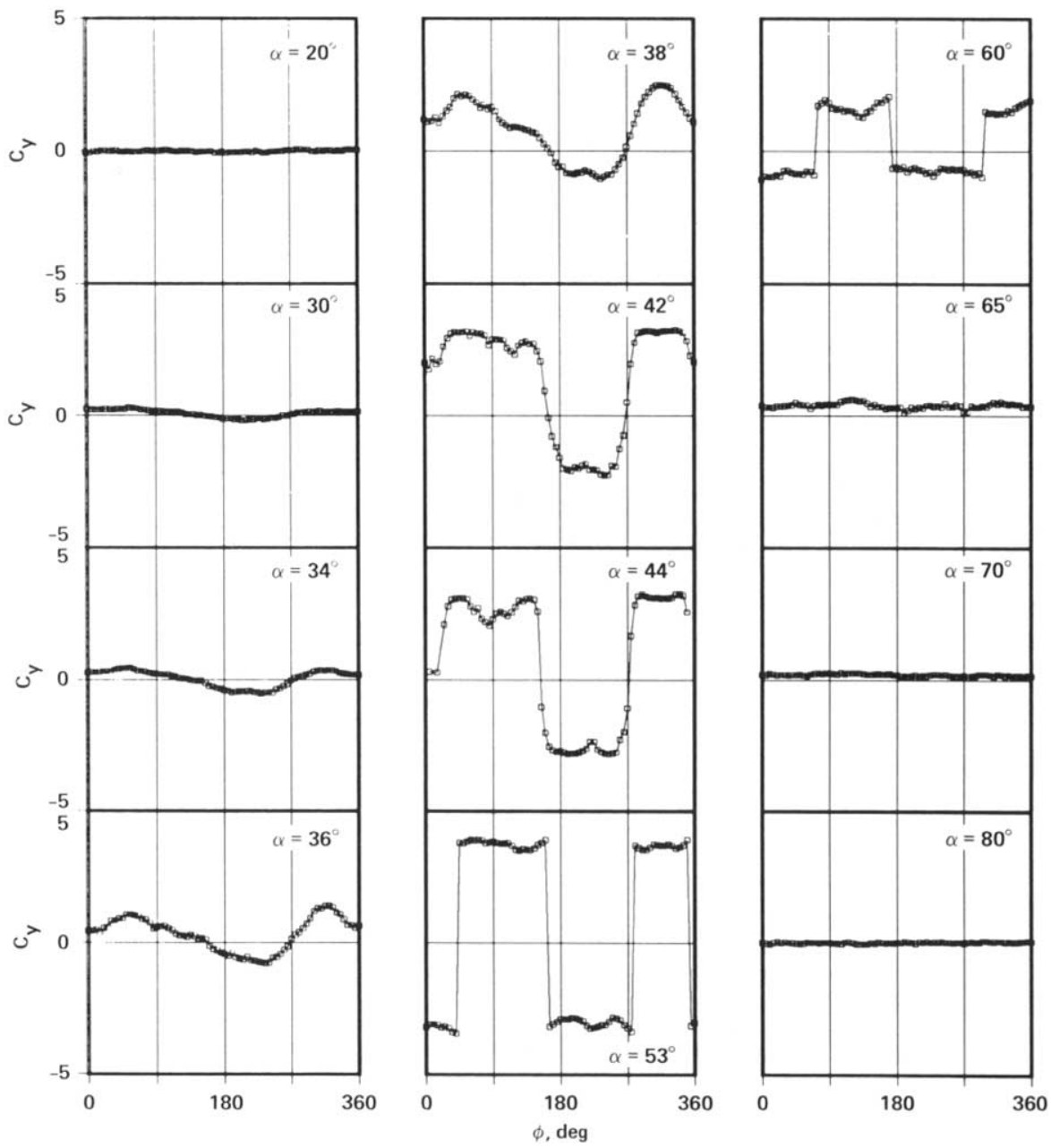

Fig. 11. Side force variation with nose roll angle (from [37]). C 1991 by the American Institute of Aeronautics and Astronautics, Inc. Reprinted with permission.

algorithms were also found to yield symmetric flowfields at high angles of attack, and were shown to be symmetric algorithms.

In order to speed up these algorithms, a diagonalization process [42] was developed that requires five scalar tridiagonal matrix inversions, and thus has much lower memory requirements and lower operation count per grid point. When the diagonal algorithm was used to compute high angle of attack flowfields, however, the results were very interesting (see Fig. 12). While the fluxsplit algorithm maintained symmetry throughout the computations, the diagonal algorithm yielded unsteady, asymmetric results. There was no obvious asymmetry in the diagonal algorithm, but after deconstructing the algorithm, Levy et al. found that the circumferential Jacobian matrix was not symmetric along the leeward plane of symmetry [39] - the algorithm was causing the flowfield asymmetry. While some researchers are content

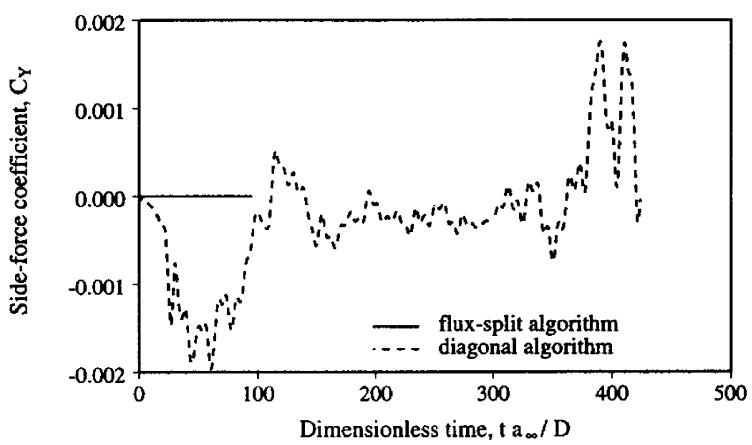

Fig. 12. Comparison of flowfield results for flux-split and diagonalized algorithms (from [39]).

to allow the algorithm to supply the perturbation that must be there anyway (from a physical perspective), it is much more desirable to use an algorithm that does not 


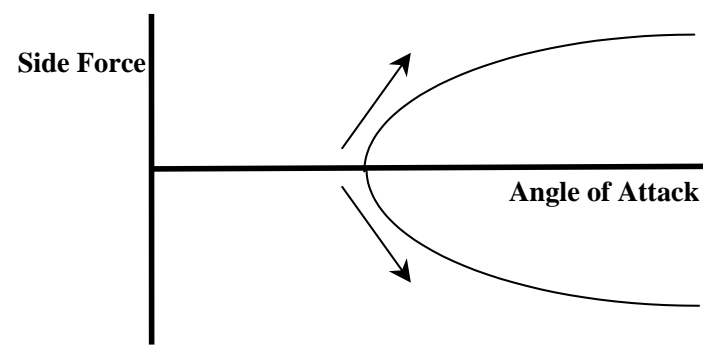

(a) bifurcation - absolute hydrodynamic instability

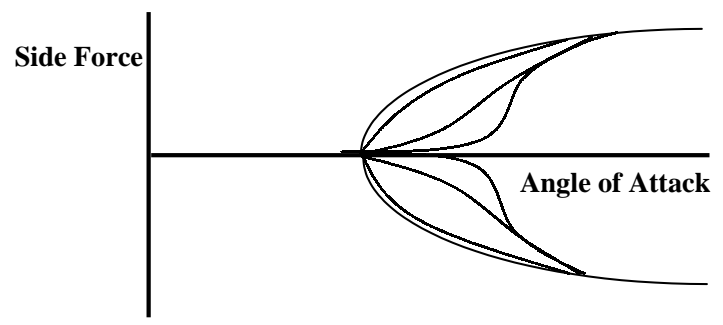

(b) unstable bifurcation convective instability

Fig. 13. Alternative explanations for high angle of attack asymmetry.

add an unknown level of perturbation. It would be superior to have the perturbation be added explicitly as a geometric disturbance or flowfield disturbance.

But there is more to these results than first meets the eye. In fact, the two views of asymmetry may have more in common than first appears. When viewed from a static angle of attack perspective, the absolute instabiliy concept and the convective instability concept are quite different (see Fig. 11). The absolute hydrodynamic stability hypothesis states that as the angle of attack is increased, a bifurcation will take place at a critical angle of attack that will produce one of two "mirror image" asymmetries (see Fig. 13a). At any angle of attack above the critical value, only two values of side force are possible; no intermediate values of side force are possible with this hypothesis. But the results of Fig. 11 and [37] show that there are angles of attack where a variety of side forces are possible.

The convective instability hypothesis states that any level of asymmetry is possible at angles of attack in the high alpha range. As the angle of attack is increased, an unstable state is reached where an infinite number of "paths" are possible until the very high angle of attack regime is reached. At that point the flowfield has reached the full bifurcated state, but in the intermediate region (high angle of attack region), there are many possible states (see Fig. 13b), which could be called an unstable bifurcation [43]. This hypothesis would explain the results found in Fig. 11, while still acknowledging that the flowfield eventually (in a pitch-up sense) is bifurcated.

\subsection{Grid generation and density}

All CFD solutions require appropriate grids, with sufficient grid density in regions of high flow gradients. The problem with accomplishing this is knowing a priori where the regions of high gradients are located. In a vortical flowfield, high gradient regions exist in the boundary layers, regions of shear layer separation, and the high-vorticity regions of the primary and secondary vortices. While all CFD solutions should be accompanied by a grid resolution study, it is common to place large numbers of grid points in regions where flow gradients are small, resulting in unnecessarily long computation times. While it is not possible to perfectly define the grid required for a good computation, some rules of thumb have been found to give reasonable results.

Many researchers have found that adequate resolution of the boundary layer is the first place to look when insuring that a grid will properly resolve a flowfield. Boundary layers require at least 20 grid point normal to the surface, with at least two of those grid points being in the laminar sub-layer [44]. Of course, more grid resolution in the boundary layer is always desired, but this normal grid resolution usually yields acceptable results. In addition, grid stretching in the normal direction should be kept below $25 \%$ increase in grid size moving away from the surface. Other definitions of grid "goodness" are available and should be investigated for high angle of attack computations [45].

Another very important grid requirement has to do with the grid resolution in the circumferential direction in the vicinity of the separation lines on the body surface. The primary and secondary separation locations require "enough" grid points for proper resolutionstudies have shown that at least five grid points should exist in the circumferential direction between separation points. Of course, this means that the same grid may not be satisfactory for different angles of attack, as the separation points move in the circumferential direction. The usual solution to this is to have a circumferential grid fineness that is high enough for all computations being performed using the grid. Obtaining a "fast" solution is usually dangerous - spending the time necessary to develop a good grid will save a great deal of work later on.

One way to create a good grid, and still not take too much time is to use multiple, overset grids [46] or unstructured grids. Overset grids can be especially useful in high angle of attack flowfield prediction as they allow the placement of high grid density in regions of high flow gradient without having to re-create the entire grid. This can save time when several calculations are being performed at different angles of attack, where the separated flow region moves to different locations in the flowfield. Other grid generation concepts should be 
developed to aid in reducing the considerable time that grid generation requires. An example of such a valuable grid generation tool is adaptive mesh refinement $[47,48]$.

\subsection{Numerical dissipation}

Numerical solutions always have some type of artificial viscosity or numerical dissipation, either explicitly or implicitly added. While implicit dissipation is the preferred method in many modern algorithms, the downside of implicit dissipation is the inability to control the level of dissipation added, even though the levels are usually quite low. The ease of use of implicit dissipation should not allow the user to be deceived into thinking that the dissipation levels will not impact the solutions.

High angle of attack flows are especially sensitive to artificially dissipation, perhaps more than many other flowfields. When the prediction of separation lines, including secondary or even tertiary separation lines, is an essential aspect of the flowfield prediction, it may be more satisfying to use explicitly added, fourth-order, dissipation. Solutions usually require fairly high levels of dissipation at the early iteration stages, but once the flowfield has settled down, the artificial dissipation should be reduced to the smallest possible levels. While it may be tempting to turn the artificial dissipation to extremely low levels, care should be taken to insure that pressure oscillations do not occur near the body surface - these oscillations can have a negative impact on the solution. A numerical experiment of dissipation levels can be found in [44] - investigations such as this should be conducted in all high angle of attack calculations. Another possible approach would be to use explicitly added viscosity and accounts for its effects [49].

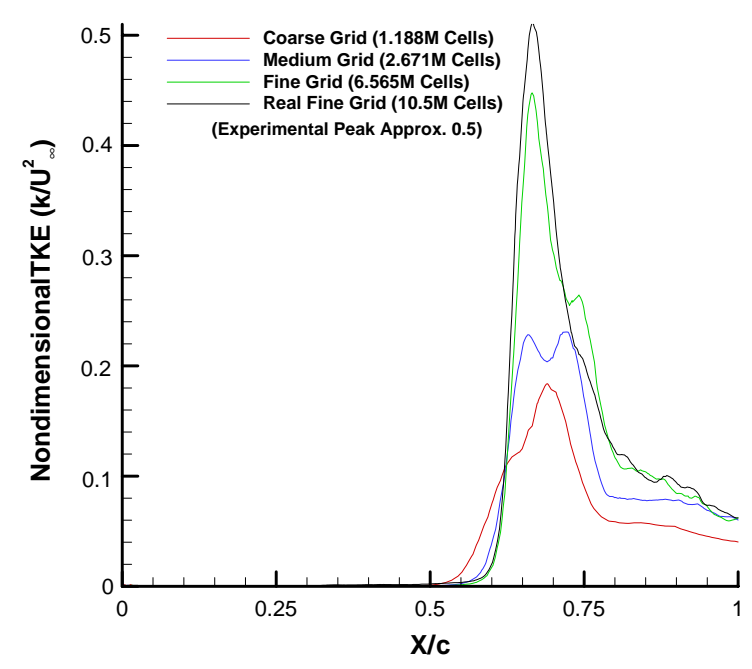

Fig. 15. Resolved turbulent kinetic energy in the vortex core for a $70^{\circ}$ delta wing at $27^{\circ}$ angle of attack.

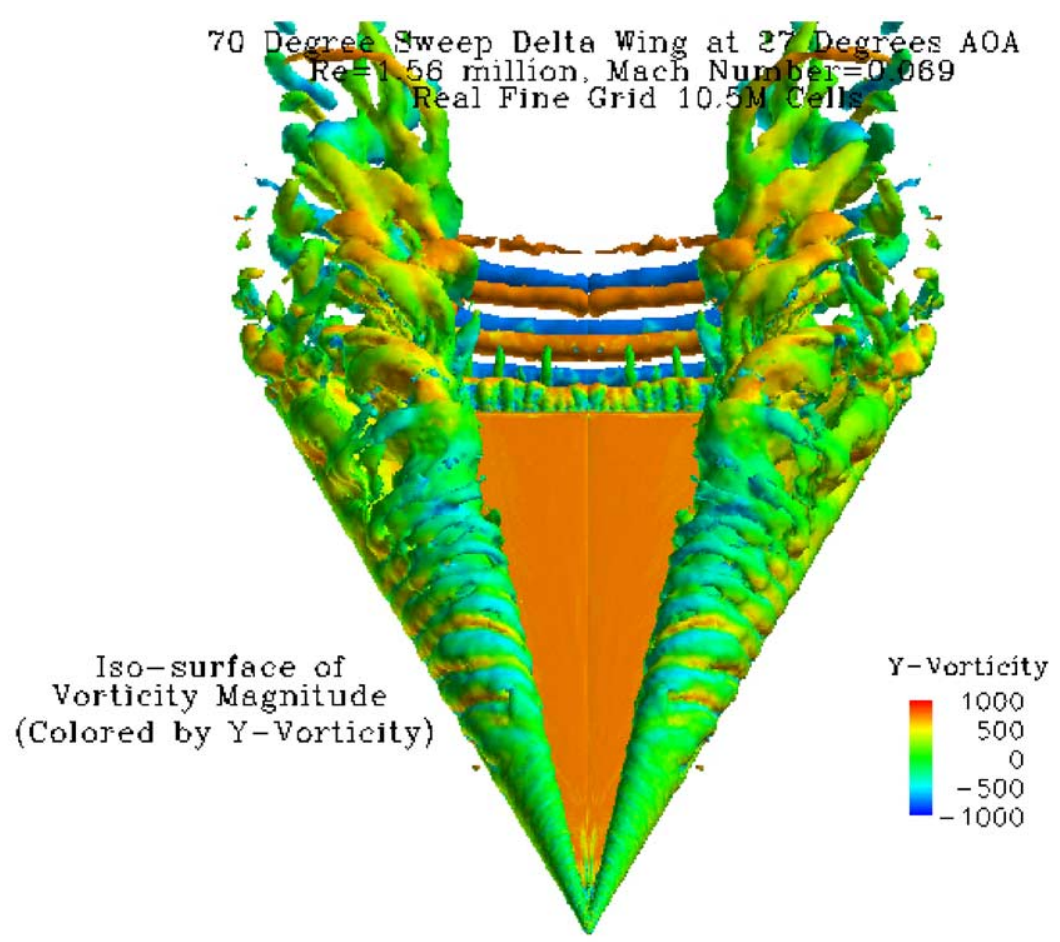

Fig. 14. Delta Wing with $70^{\circ}$ of sweep at $27^{\circ}$ angle of attack (from [50]). 


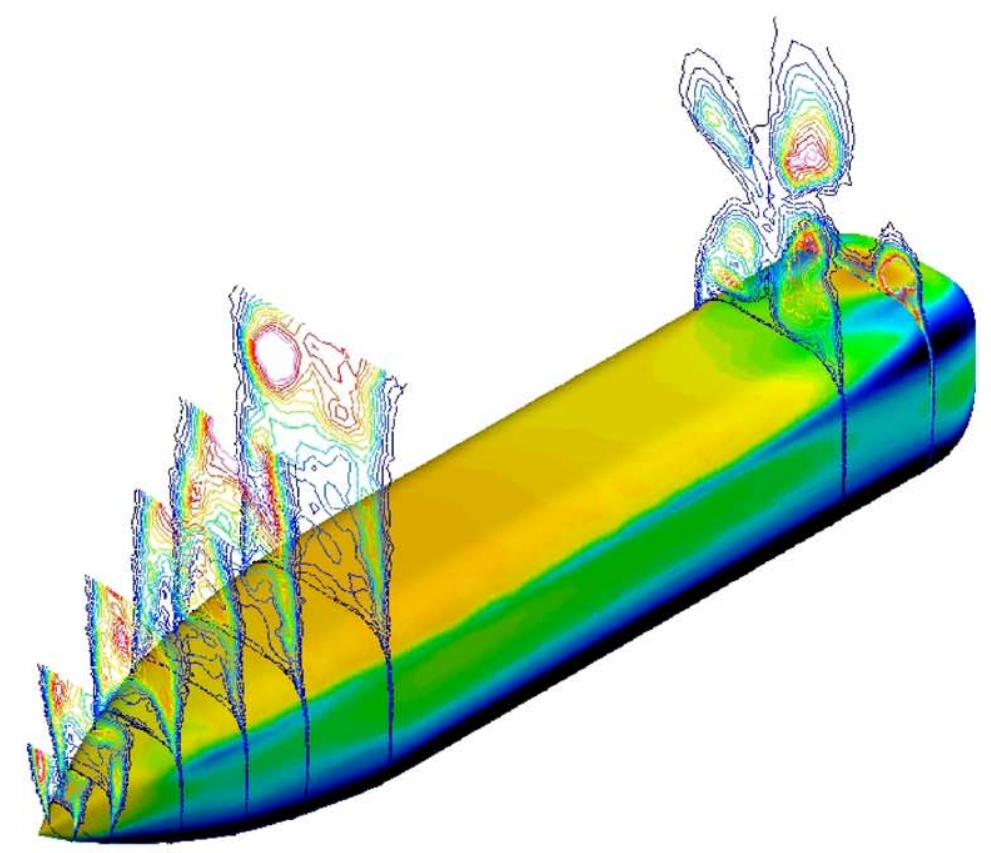

(a) Vorticity contours, $\mathrm{x} / \mathrm{L}=0.222$

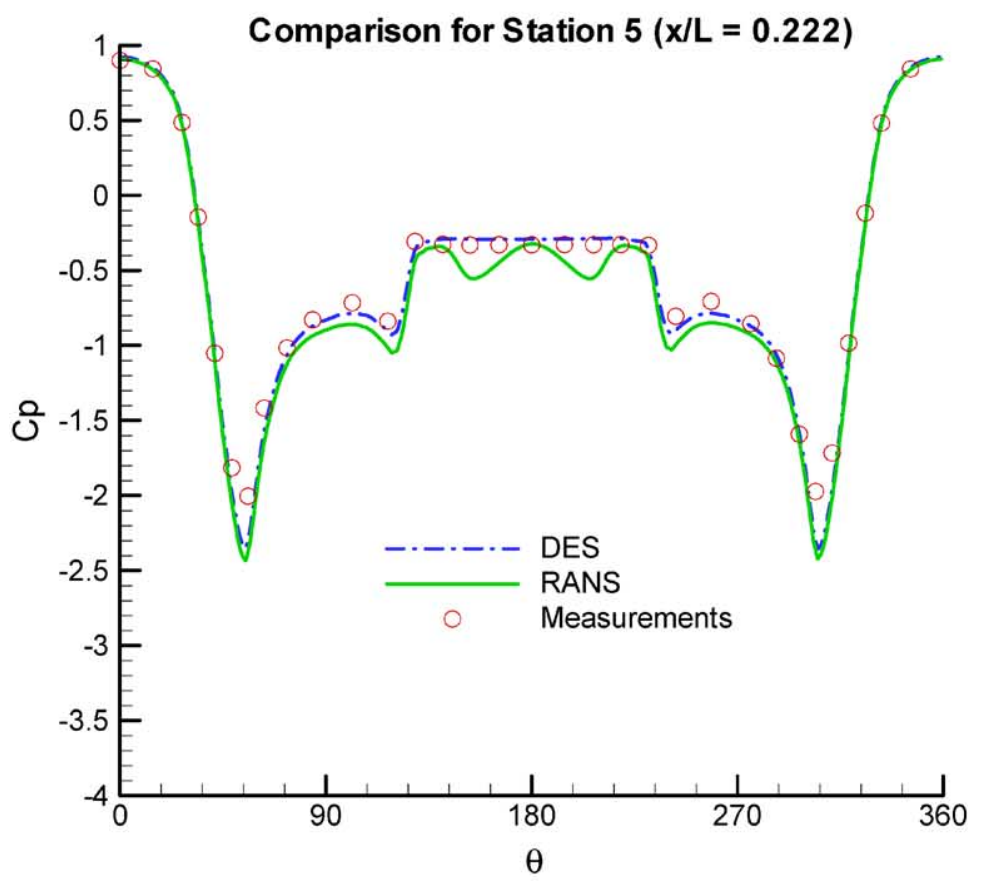

(b) Surface pressures, $\mathrm{x} / \mathrm{L}=0.222$

Fig. 16. 6:1 rectangular ogive at $90^{\circ}$ angle of attack (from [52]).

\section{Computational results and future directions}

Recent computations using DES have shown great promise for predicting massively separated flowfields. A detailed numerical evaluation of the flow over a delta wing at high angles of attack shows incredible detail in the flow [50]. Fig. 14 shows the delta wing flowfield where the shear layer instability along the leading edge is 


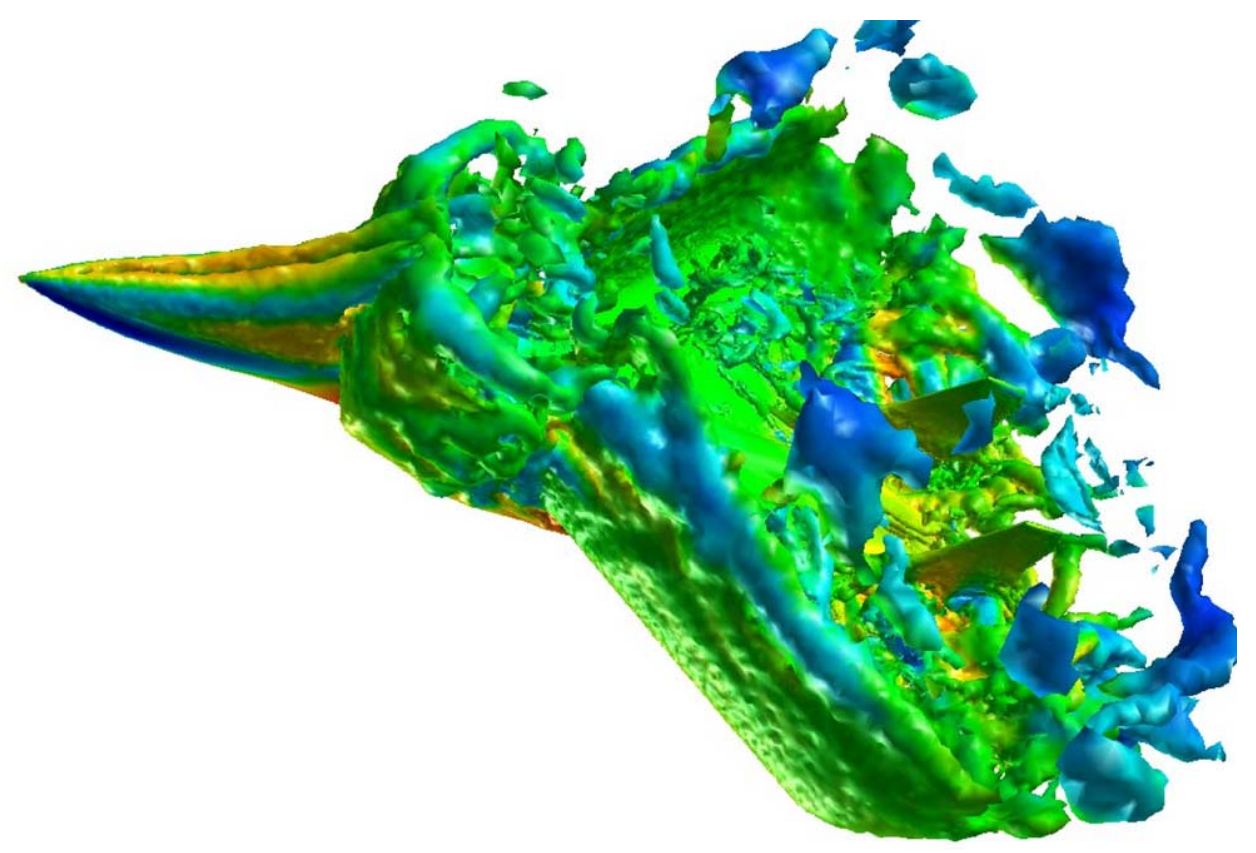

(a) Iso-surfaces of vorticity over the aircraft

\begin{tabular}{|c|c|c|c|c|c|c|c|}
\hline & & $\mathbf{C}_{\mathrm{L}}$ & $\mathbf{C}_{\mathrm{D}}$ & $\mathrm{C}_{\mathrm{M}}$ & ${ } \mathrm{C}_{\mathrm{L}}$ & $\% \mathrm{C}_{\mathrm{D}}$ & ${ } \mathrm{C}_{\mathrm{M}}$ \\
\hline & Exp & 0.781 & 1.744 & -0.466 & & & \\
\hline & Coarse & 0.747 & 1.677 & -0.431 & $-4.25 \%$ & $3.86 \%$ & $-7.62 \%$ \\
\hline DES & Baseline & 0.736 & 1.616 & -0.495 & $-5.70 \%$ & $-7.35 \%$ & $6.10 \%$ \\
\hline & Fine & 0.759 & 1.648 & -0.457 & $-2.81 \%$ & $-5.52 \%$ & $-2.00 \%$ \\
\hline & Coarse & 0.855 & 1.879 & -0.504 & $9.49 \%$ & $7.73 \%$ & $8.17 \%$ \\
\hline S-A & Baseline & 0.852 & 1.867 & -0.523 & $9.09 \%$ & $7.05 \%$ & $12.22 \%$ \\
\hline & Fine & 0.860 & 1.880 & -0.507 & $10.22 \%$ & $7.78 \%$ & $8.72 \%$ \\
\hline
\end{tabular}

(b) Comparisons with experimental data

Fig. 17. F-15E at $65^{\circ}$ angle of attack (from [53]); DES - detached-eddy simulation, S-A - Spalart-Almaras turbulence model.

clearly evident, as well as vortex breakdown, and shear layer roll-up from the delta wing blunt base. Computations of this complexity are not possible with RANS calculations, since the unsteady flow features would not be able to be resolved using time-averaged turbulence models.

Fig. 15 displays the resolved turbulent kinetic energy along the vortex core compared with experimental data [51]. As the grid is resolved the experimental peak is reached, although the computation required 10.5 million unstructured cells to attain the experimental level of resolved turbulent kinetic energy.

Another recent application of DES for a massively separated flowfield is a $6: 1$ rectangular ogive at $90^{\circ}$ angle of attack (see Fig. 16a; the forebody cross-section is shown in Fig. 10) [52]. This flowfield challenges RANS models because they are unable to properly resolve the pressure variations on the leeward side of the body. Even with modifications such as Degani-Schiff, the results for RANS models are still "averaged", and the averaging process does not allow for true unsteadiness to develop in the separated flow region. When DES is applied to the flowfield, the unsteady movement of the vortical structures "washes out" the pressures on the leeward surface, giving a flat pressure profile that matches experimental data, as shown in Fig. 16b.

The end goal of massively separated flow computations is to accurately predict aircraft at high angles of 
attack, including post-stall flowfields. Shown in Fig. 17 is the F-15E at $65^{\circ}$ angle of attack modeled as a half body with nearly 6 million cells [53]. Iso-surfaces of vorticity are shown, and comparisons with available experimental data reveal that the results are within $6 \%$ for lift, drag, and pitch moment coefficients. Of course, the aircraft would have to be resolved with both left and right sides in order to obtain the asymmetric flowfields that are certainly occurring, but the ability of DES to capture the complexities of this massively separated flowfield are impressive.

While these DES results are impressive, they do not represent a final stage of high angle of attack flow prediction. Researchers will need to continue to investigate these types of hybrid RANS-LES models and insure that they work well for a wide variety of separated, vortical flowfields. In spite of this, however, the current state of CFD prediction for high angle of attack flowfields has progressed significantly, with the accurate prediction of full-scale maneuvering aircraft at hand.

\section{Conclusions}

High angle of attack flow computations have a variety of unusual aspects that make accurate predictions challenging. A variety of influences on high angle of attack flow predictions have been discussed, including: governing equation complexity, turbulence modeling, transition modeling, algorithm symmetry, grid generation and density, and numerical dissipation. While some of these issues are important in many flowfield calculations, successful simulation of high angle of attack flowfields must consider all of these factors. It is very easy to get a poor solution for these highly separated, vortical flowfields! A little careful forethought, planning, and evaluation can lead to amazingly useful simulations. Researchers should realize that when it comes to high angle of attack flow predictions, faster is rarely betterspend extra time in the beginning of the simulation work and very good results are obtainable.

\section{References}

[1] Keener ER, Chapman GT. Similarity in vortex asymmetries over slender bodies and wings. AIAA J 1977;15: 1370-2.

[2] Rom J. High angle of attack aerodynamics. New York: Springer, 1992.

[3] Ericsson LE, Reding JP. Asymmetric vortex shedding from bodies of revolution. In: Hemsch MJ, Nielsen JN, editors. Tactical missile aerodynamics. New York: American Institute of Aeronautics and Astronautics, 1986.

[4] Bertin JJ. Aerodynamics for engineers. Upper Saddle River, NJ: Prentice-Hall, 2002.
[5] Van Dyke M. An album of fluid motion. Stanford: The Parabolic Press, 1982.

[6] Degani D, Schiff LB. Computation of turbulent flows around pointed bodies having crossflow separation. J Comput Phys 1986;66(1):173-96.

[7] Degani D, Levy Y. Asymmetric turbulent vortical flows over slender bodies. AIAA J 1992;30(9):2267-73.

[8] Hummel D. On the vortex formation over a slender wing at large angles of incidence. In: High Angle of Attack Aerodynamics, AGARD Conference Proceedings CP-247, 1979.

[9] Viviand H. Conservation forms of gas dynamics equations. La Recherche Arospatiale 1974;1:65-6.

[10] Moin P. Advances in large eddy simulation methodology for complex flows. Int J Heat Fluid Flow 2002;23: 710-20.

[11] Spalart PR. Strategies for turbulence modeling and simulations. Proceedings of the Fourth International Symposium on Engineering Turbulence Modeling and Measurements. Amsterdam: Elsevier Science, 1999. p. 3-17.

[12] Spalart PR, Jou W-H, Strelets M, Allmaras SR. Comments on the feasibility of LES for wings, and on a hybrid RANS/LES approach. Advances in DNS/LES, First AFOSR International Conference on DNS/LES, Rusliton, CA, Greyden Press, Columbus, 1997.

[13] Shur M, Spalart PR, Strelets, M, Travin A. Detached-eddy simulation of an airfoil at high angle of attack. Proceedings of the Fourth International Symposium on Engineering Turbulence Modeling and Measurements. Amsterdam: Elsevier Science, 1999. p. 669-78.

[14] Degani D, Marcus SW. Thin vs. full Navier-Stokes computation for high-angle-of-attack aerodynamics. AIAA J 1997;35(3):565-7.

[15] Reynolds O. On the dynamical theory of incompressible viscous fluids and the determination of the criterion. Philos Trans. R Soc London Ser A 1895;186:123-64.

[16] Favre A. Equations des gaz turbulents compressibles. J Mc 1965;4:361-90.

[17] Reynolds WC. Computation of turbulent flows. Annu Rev Fluid Mech 1976;8:183-208.

[18] Wilcox DC. Turbulence modeling for CFD, 2nd ed.. LaCañada, CA: DCW Industries, 2002.

[19] Baldwin B, Lomax H. Thin-layer approximation and algebraic model for separated turbulent flows. AIAA Paper 78-257, January 1978.

[20] Hartwich PM, Hall RM. Navier-Stokes solutions for vortical flows over a tangent-ogive cylinder. AIAA J 1990; 28(7):1171-9.

[21] Vatsa VN. Viscous flow solutions for slender bodies of revolution at incidence. Comput Fluids 1991;23(3):313-20.

[22] Gee K, Cummings RM, Schiff LB. Turbulence model effects on separated flow about a prolate spheroid. AIAA J 1992;30(3):655-64.

[23] Dacles-Mariani J, Zilliac GG, Chow JS, Bradshaw P. Numerical/experimental study of a wingtip vortex in the near field. AIAA J 1995;33(9):1561-8.

[24] Josyula E. Computational simulation improvements of supersonic high-angle-of-attack missile flows. J Spacecr Rockets 1999;36(1):59-66.

[25] Murman, SR. Vortex filtering for turbulence models applied to crossflow separation. AIAA Paper 2001-0114, January 2001. 
[26] Spalart PR, Allmaras SR. A one-equation turbulence model for aerodynamic flows. AIAA Paper 92-0439, January 1992.

[27] Smagorinski J. General circulation experiments with the primitive equations. Mon Weather Rev 1963;91:261-341.

[28] Strelets M. Detached eddy simulation of massively separated flows. AIAA Paper 2001-0879, January 2001.

[29] Forsythe JR, Hoffmann KA, Cummings RM, Squires KD. Detached-eddy simulation with compressibility corrections applied to a supersonic axisymmetric base flow. J Fluids Eng 2002;124(4):911-23.

[30] Polhamus EC, Geller EW, Grunwald KJ. Pressure and force characteristics of noncircular cylinders as affected by Reynolds number with a method included for determining the potential flow about arbitrary shapes. NASA Technical Report R-46, 1959.

[31] Squires KD, Forsythe JR, Spalart PR. Detached-eddy simulation of the separated flow around a forebody crosssection. In: Geurts BJ, Friedrich R, Métais O, editors. Direct and large-eddy simulation IV. Dordrecht: Kluwer Academic Publishers, 2001. p. 481-500.

[32] Travin A, Shur M, Strelets M, Spalart P. Detached-eddy simulations past a circular cylinder. Flow Turbulence Combust 2000;63(1):293-313.

[33] Arnal D, Casalis G. Laminar-turbulent transition prediction in three-dimensional flows. Progr Aerospace Sci 2000;36(2):173-91.

[34] Stock HW, Haase W. Feasbility Study of $e^{N}$ transition prediction in Navier-Stokes methods for airfoils. AIAA J 1999;37(10):1187-96.

[35] Crouch JD, Crouch IWM, Ng LL. Transition prediction for three-dimensional boundary layers in computational fluid dynamics applications. AIAA J 2002;40(8):1536-41.

[36] Thomas JL. Reynolds number effects on supersonic asymmetrical flows over a cone. J Aircr 1993;30(4):488-95.

[37] Zilliac GG, Degani D, Tobak M. Asymmetric vortices on a slender body of revolution. AIAA J 1991;29(5):667-75.

[38] Luo SC, Lua KB, Goh EKR. Side force on an ogive cylinder: effects of surface roughness. J Aircr 2002;39(4): 716-8.

[39] Levy Y, Hesselink L, Degani D. Anomalous asymmetries in flows generated by algorithms that fail to conserve symmetry. AIAA J 1995;33(6):999-1007.
[40] Beam RM, Warming RF. An implicit finite-difference algorithm for hyperbolic systems in conservation law form. J Comput Phys 1976;22(9):87-110.

[41] Steger JL, Warming RF. Flux vector splitting of the inviscid gasdynamic equations with applications to finite-difference methods. J Comput Phys 1981;40(2): 263-93.

[42] Pulliam TH, Chausee DS. A diagonal form of an implicit approximate-factorization algorithm. J Comput Phys 1981;39(2):347-63.

[43] Huerre P. private communication.

[44] Schiff LB, Degani D, Cummings RM. Computation of three dimensional turbulent vortical flows on bodies at high incidence. J Aircr 1991;28(10):689-99.

[45] Thompson J, Matlin C, Gatlin B. Analysis and control of grid quality in computational simulation. Wright Laboratory TR-91-83, June 1992.

[46] Cummings RM, Rizk YM, Schiff LB, Chaderjian NM. Simulation of high-incidence flow about the F-18 fuselage forebody. J Aircr 1992;29(11):565-74.

[47] Pirzadeh SZ. Vortical flow prediction using an adaptive unstructured grid method. NATO Research \& Technology Organization, Applied Vehicle Technology Panel Meeting, Norway, 7-11 May 2001.

[48] Mitchell A, Morton S, Forsythe J. Analysis of delta wing vortical substructures using detached-eddy simulation. AIAA Paper 2002-2968, June 2002.

[49] Varma RR, Caughey DA. Evaluation of Navier-Stokes solutions using the integrated effect of numerical dissipation. AIAA J 1994;32(2):294-300.

[50] Morton SA, Forsythe JR, Mitchell AM, Hajek D. Detached-eddy simulations and Reynolds-averaged Navier-Stokes simulations of delta wing vortical flowfields. J Fluids Eng 2002;124(4):924-32.

[51] Mitchell AM, Molten P. Vortical substructures in the shear layers forming leading-edge vortices. AIAA J 2002;40(8): 1689-92.

[52] Viswanathan A, Klismith K, Forsythe JR, Squires KD. Detached-eddy simulation around a rotating forebody, AIAA Paper 2003-0263, January 2003.

[53] Forsythe JR, Squires KD, Wurtzler KE, Spalart PR. Detached-eddy simulation of fighter aircraft at high alpha, AIAA Paper 2002-0591, January 2002. 